\title{
Meta Cookie+: An Illusion-Based Gustatory Display
}

\author{
Takuji Narumi $^{1}$, Shinya Nishizaka ${ }^{2}$, Takashi Kajinami ${ }^{2}$, \\ Tomohiro Tanikawa ${ }^{2}$, and Michitaka Hirose ${ }^{2}$ \\ ${ }^{1}$ Graduate School of Engineering, The University of Tokyo / JSPS \\ 7-3-1 Hongo Bunkyo-ku, Tokyo Japan \\ ${ }^{2}$ Graduate School of Information Science and Technology, The University of Tokyo \\ 7-3-1 Hongo Bunkyo-ku, Tokyo Japan \\ narumi@cyber.t.u-tokyo.ac.jp, \\ \{nshinya, kaji, tani, hirose\}@cyber.t.u-tokyo.ac.jp
}

\begin{abstract}
In this paper, we propose the illusion-based "Psuedo-gustation" method to change perceived taste of a food when people eat by changing its appearance and scent with augmented reality technology. We aim at utilizing an influence between modalities for realizing a "pseudo-gustatory" system that enables the user to experience various tastes without changing the chemical composition of foods. Based on this concept, we built a "Meta Cookie+" system to change the perceived taste of a cookie by overlaying visual and olfactory information onto a real cookie. We performed an experiment that investigates how people experience the flavor of a plain cookie by using our system. The result suggests that our system can change the perceived taste based on the effect of the cross-modal interaction of vision, olfaction and gustation.
\end{abstract}

Keywords: Illusion-based Virtual Reality, Gustatory Display, Pseudogustation, Cross-modal Integration, Augmented Reality.

\section{Introduction}

Because it has recently become easy to manipulate some kind of multimodal information by using a computer, many research projects have used computergenerated virtual reality for studying the input and output of haptic and olfactory information in order to realize more number of realistic applications [1]. However, few of these studies have dealt with gustatory information, and there have been few display systems presenting gustatory information [2,3].

This scarcity of research on gustatory information is for several reasons. One reason is that taste sensation is based on chemical signals, whose functions have not yet been fully understood. Another reason is that taste sensation is affected by other factors such as vision, olfaction, thermal sensation, and memory. Thus, as described above, the complexity of the cognition mechanism for gustatory sensation makes it difficult to build up a gustatory display, which is able to present a wide variety of gustatory information.

Our hypothesis is that the complexity of the gustatory system can be applied to the realization of a pseudo-gustatory display that presents the desired flavors by means of 
a perceptual illusion. The cases I have in mind are ones in which what you sense with one modality affects what you experience in another. For example, the ventriloquist effect involves an illusory experience of the location of a sound that is produced by the sound's apparent visible source. The effect is neither inferential nor cognitive, but results from cross-modal perceptual interactions. Cross-modal interactions, however, are not limited to vision's impact upon the experience through other sense modalities. By using this illusionary effect, we may induce people to experience different flavors when they taste the same chemical substance. Therefore, in order to realize a novel gustatory display system, we aim to establish a method for eliciting and utilizing a cross-modal interaction.

In this paper, we propose a method to change the perceived taste of a cookie when it is being eaten based on an illusion evoked by changing its appearance and scent by employing augmented reality technology. We then report a "Meta Cookie" system, which implements the proposed method, and the results of an experiment that investigates how people experience the flavor of a plain cookie by using our system.

\section{Cross-Modal Interactions Underlying the Perception of Flavor}

Fundamental tastes are considered the basis for the presentation of various tastes similar to how basic colors such as RGB are used as the basis for visual systems. According to physiological definitions, taste has the status of a minor sense, as the channel of only a limited number of sensations: sweetness, sourness, bitterness, saltiness, and umami [4].

What is commonly called taste signifies a perceptual experience that involves the integration of various sensations. When we use the common word "flavor" in place of taste, then, we are again referring to what is a quite multi-faceted sensation. In fact, the International Standards Organization has defined flavor as a complex combination of the olfactory, gustatory, and trigeminal sensations perceived during tasting [5]. Auvray et al. reviewed the literature on multisensory interactions underlying the perception of flavor; furthermore, they summarized saying that flavor is not defined as a separate sensory modality but as a perceptual modality that is unified by the act of eating, and should be used as a term to describe the combination of taste, smell, touch, visual cues, auditory cues, and the trigeminal system [6]. These definitions suggest that it is possible to change the flavor that people experience from foods by changing the feedback they receive through modalities other than the sense of taste. While it is difficult to present various tastes through a change in chemical substances, it is possible to induce people to experience various flavors without changing the chemical ingredients but by changing only the other sensory information that they experience.

The sense of smell is most closely related to our perception of taste above all other senses. This relationship between gustatory and olfactory sensations is commonly known, as illustrated by pinching our nostrils when we eat food that we find displeasing. Indeed, it has been reported that most of what people commonly think of as the taste of food actually originates from the nose [7]. Conversely, another set of researches on taste enhancement has provided strong support for the ability of odors 
to modify taste qualities [8]. These studies indicate the possibility of changing the flavor that people experience with foods by changing the scent.

Conversely, under many conditions, it is well known that humans have a robust tendency to rely upon vision more than other senses. Several studies have explored the effect of visual stimuli on our perception of flavor. For instance, taste and flavor intensity have been shown to increase as the color level in a solution increases [9]. However, Spence et al. state the empirical evidence regarding the role that food coloring plays in the perception of the intensity of a particular flavor or taste that is attributed (as reported by many researchers over the last 50 years), which is rather ambiguous because food coloring most certainly influences people's flavor identification responses [10]. Their survey suggests the possibility of changing the flavor identification by changing the appearance of food.

Therefore, our research focuses on the technological application of the influence of appearance and scent on flavor perception. We propose a method to change the perceived taste of food by changing its appearance and scent. In this study, we use cookies as an example application. This is because cookies have a wide variety of appearance, scent, and taste; however, at the same time, almost all cookies are similar in texture and shape. Thus, we have developed a system to overlay the appearance and scent of a flavored cookie on a plain cookie to let users experience eating a flavored cookie although they are just eating a plain cookie.

\section{Pseudo-gustatory Display: MetaCookie+}

We developed a system, which we have named "MetaCookie+," to change the perceived taste of a cookie by overlaying visual and olfactory information onto a real cookie with a special AR marker pattern.

\subsection{System Overview}

"MetaCookie+" (Fig. 1) comprises four components: a marker-pattern-printed plain cookie, a cookie detection unit based on an Edible Marker System, an overlaying visual information unit, and an olfactory display. Fig. 2 illustrates the system configuration of "MetaCookie+." Each component is discussed in more detail in the following sections.

In this system, a user wears a head-mounted visual and olfactory display system. The cookie detection unit detects the marker-pattern-printed cookie and calculates the state (6DOF coordinate/occlusion/division/distance between the cookie and the nose of a user) of the cookie in real time. Based on the calculated state, an image of a flavored cookie is overlaid onto the cookie. Moreover, the olfactory display generates the scent of a flavored cookie with an intensity that is determined based on the calculated distance between the cookie and the nose of a user. The user can choose one cookie, which s/he wants to eat, from multiple types. The appearance and scent of the flavored cookie, which the user selects, are overlaid onto the cookie. 


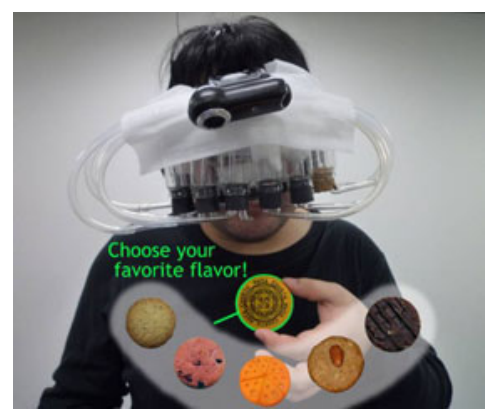

Fig. 1. MetaCookie+

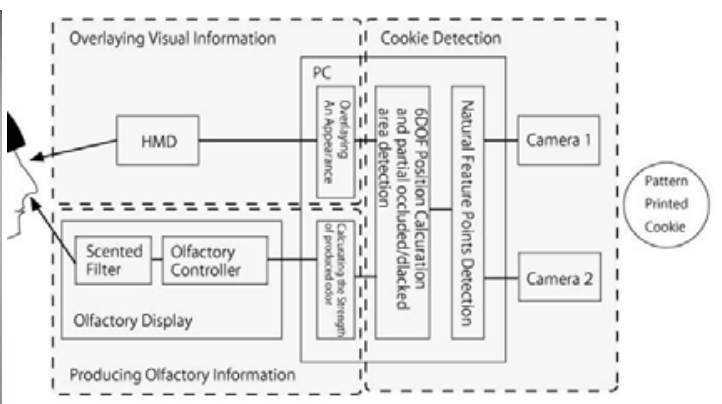

Fig. 2. System configuration of "MetaCookie+"

\subsection{Edible Marker System}

For taste augmentation, an interaction with foods is necessary. At the time when the foods must be eaten or divided, a method to detect occlusion and division is required. However, conventional object-detection methods assume that a target object is continuous. When the object is divided into pieces, tracking will fail because the feature points of only a single piece are recognized as the target object whereas other feature points are regarded as outliers. Despite the importance of division as one of the state changes of an object, it has not been studied from the viewpoint of object detection for AR applications.

Therefore, we proposed the "Edible Marker" system, which not only estimates the 6DOF coordinate of the AR marker, but also detects its occlusion and division. We then applied this system to "MetaCookie+." Fig. 3 shows the processing steps of the occlusion- and division-detectable "Edible Marker" system. The Edible Marker System estimates the 6DOF coordinate, occlusion, and division of a marker in three steps: Marker Detection, Background Subtraction and Superimposition.

In step 1 (Marker Detection), the natural feature points are detected from the captured image and the marker position is extracted from an estimated homography matrix. Subsequently, the projected image of the marker area in the captured image can be obtained. For implementation, we used Fern [11] as the natural feature descriptor and classifier.

In a conventional planar-object detection method, a homography matrix is estimated from the correspondence of the feature points between a prepared template image and an image captured by the user's camera. An accurate homography matrix is estimated by calculating its elements using the least squares method after outlier elimination. If we simply apply the conventional method to a divided planar object, only one out of all the pieces of the object is detected as an inlier; therefore, the other pieces cannot be detected as parts of the object. To detect all pieces of the divided object, another method is required. The proposed method detects the pieces of the divided object by iteratively applying PROSAC [12]. A database of the target object's feature points is prepared in advance. In each estimation process, the inlier points are deleted from the database. Next, estimation is performed using the updated database. Subsequently, this method detects the inliers for each piece of the target object. The iteration stops when the homography matrix calculation fails. After these processes, the projected image of each piece in the captured image is obtained. 
In step 2 (Background Subtraction), a difference image is obtained by background subtraction. We implemented background subtraction based on the method shown in [13]. The image to the left of the middle panel in Fig. 3 is the difference between the template image and the projected image. Combining the temporary result and the mask image for superimposition, the final result is obtained.

In step 3 (Superimposition), the image to the left of bottom panel in Fig. 3 is overlaid using the final result obtained in step 2. The result of superimposition is shown in the image to the right.

Fig. 4 shows that the proposed object-detection system can be recognized even if the cookie is eaten, partially occluded, or divided. Furthermore, the area on which the image should be overlaid can be detected by the background subtraction method. The marker can manage an occlusion/division that is more than half of an entire cookie.
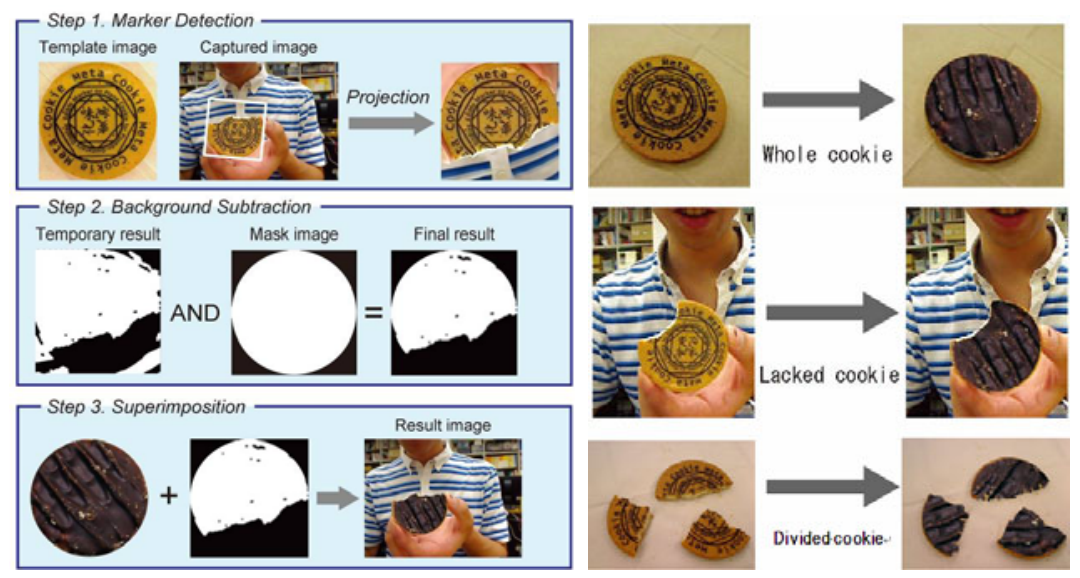

Fig. 3. Processing steps of the occlusion-and-division-detectable "Edible Marker" system and realistic superimposition based on the detection

\subsection{Pattern Printed Cookie}

We made a plain cookie detectable by a camera for this system by printing a pattern on it with a food printer. We use a MasterMind's food printer "Counver" [14], which is a commercial off-the-shelf product. The printer produces a jet of colored edible ink and creates a printed image on a flat surface of food.

\subsection{Cookie Detection and Overlaying an Appearance}

The Cookie Detection unit based on the Edible Marker system can obtain the 6DOF coordinate of the cookie and the distance between the cookie and the camera. In the Cookie Detection phase, two cameras are used in parallel. We used two Logicool Webcam Pro 9000 cameras (angle of view: $76^{\circ}$ ) in this implementation. The layout of the cameras, a head-mounted display (HMD), and an olfactory display is shown in Fig. 4. The range of the cameras is also illustrated in this figure. The two cameras are positioned to eliminate blind spots between the user's hands and mouth, in order to 
track the cookie from the time at which a user holds it to the time at which s/he puts it in her/his mouth.

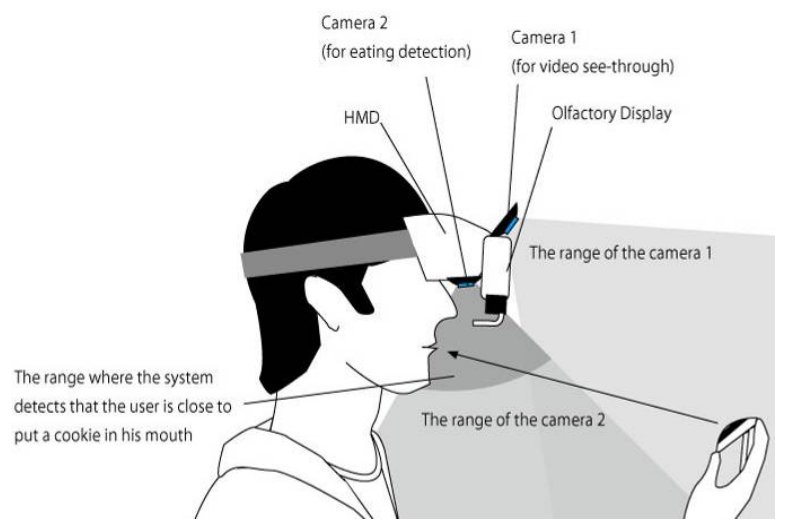

Fig. 4. Layout of two cameras, a head-mounted display and an olfactory display

Camera 1 in Fig. 6 is for overlaying the appearance of another cookie on the marked cookie and deciding the strength of the produced smell. The relationship of the distance between the cookie and the camera to the strength of the produced smell is discussed below. This camera and an HMD are used for Video see-through. The HMD displays an image of several types of cookies on the pattern-printed cookie based on the estimated position and detected occlusion/division. This visual effect allows users to experience eating a selected cookie while merely eating a plain cookie.

Another camera (Camera 2 in Fig. 6) is positioned in front of the user's nose and oriented in the downward direction in order to detect when the user eats a cookie. The area near the user's mouth is outside the first camera's line of vision. This is because we placed the second camera in front of the user's nose and oriented it in the downward direction. When the second camera detects that there is a cookie in front of the user's mouth (within $15 \mathrm{~cm}$ from the camera), the system recognizes that the user is about to put a cookie in her/his mouth.

\subsection{Olfactory Display}

We use an air-pump-type head-mounted olfactory display (Fig. 5) to produce the scent of the selected cookie. The olfactory display comprises six air pumps, a controller, and scented filters. One is to send fresh air and five pumps are to send scented air. Each pump for scented air is connected to a scent filter filled with aromatic chemicals. It can eject fresh air and six types of scented air. The scent filters add scents to air from the pumps, and the scented air is ejected near the user's nose. The strength of these scents can be adjusted to 127 different levels. By mixing fresh air and scented air, the olfactory display generates an odor in arbitrary level with same air volume. Users are unable to feel any change in air volume when the strength of the generated odor changes. 
According to the position of the pattern-printed plain cookie, the controller drives the air pumps. Nearer the marked cookie from the user's nose, stronger scent ejects from the olfactory display. Response time for generating arbitrary odor is less than 50 ms. It is quick enough to let users experience the change of smell in synchronization with the change of visual information.

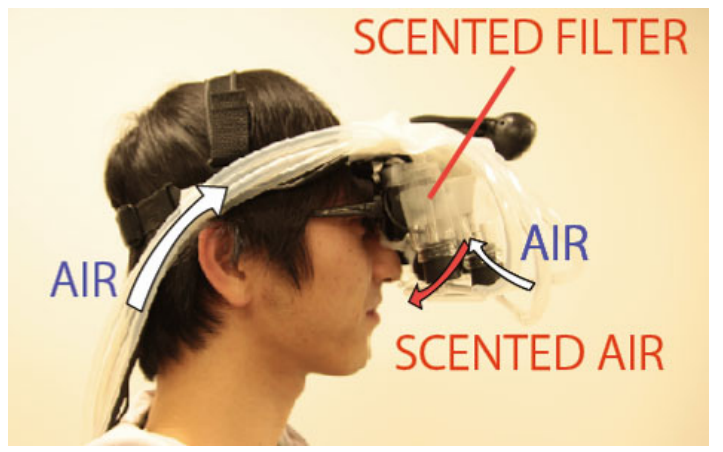

Fig. 5. Air-pump type head-mounted olfactory display

"MetaCookie+" generates two patterns of olfactory stimuli for simulating orthonasal and retronasal olfaction. One pattern simulates orthonasal olfaction and functions after the user holds the pattern-printed cookie and before s/he brings it near her/his mouth. In this pattern, the controller drives the air pumps according to the position of the pattern-printed plain cookie. The nearer the pattern-printed cookie is to the user's nose, the stronger the scent ejected from the olfactory display. The olfactory display is activated when the cookie is detected within $50 \mathrm{~cm}$ from camera 1 . The value of $50 \mathrm{~cm}$ is determined based on the average distance between the cameras and a $70 \mathrm{~cm}$-high desk along the line of sight when the user sits on a chair in front of the desk. The strength of the smell produced by the olfactory display is zero when the distance is $50 \mathrm{~cm}$ and strongest when the distance is $0 \mathrm{~cm}$. The output is controlled linearly within $50 \mathrm{~cm}$ from camera 1 .

Another pattern simulates retronasal olfaction and functions after the system recognizes that the user is about to put a cookie in her/his mouth with camera 2 . When camera 2 detects a cookie in front of the user's mouth, the system produces the strongest smell from the olfactory display for $30 \mathrm{~s}$. We determined the period to be longer than the time to finish eating a bite of the cookie.

This olfactory information evokes a cross-modal effect between olfaction and gustation, and enables users to feel that they are eating a flavored cookie although they are just eating a plain cookie.

\section{Evaluation}

In order to evaluate the effectiveness of our proposed method for inducing people to experience various flavors, we conducted an experiment to investigate how people experience flavor in a cookie by using the "Meta Cookie" system. The purpose of this 
experiment was to examine the cross-modal effect of visual stimuli and olfactory stimuli on gustation. We investigated how the participants would perceive and identify the taste of the cookie under conditions of only visual augmentation and only olfactory augmentation and visual and olfactory augmentation. We prepared two types of appearances and scents of commercially available cookies: chocolate and tea. We examined how the participants experience and identfy the taste of a plain cookie with these appearances and scent that are overlaid using our system.

\subsection{Experimental Protocol}

The combinations of scent and appearance in each experimental condition, which were used in the experiment for representing flavored cookies, are illustrated in Fig. 6. There are 7 combinations (without augmentation, visual augmentation (chocolate), visual augmentation (tea) olfactory augmentation (chocolate) olfactory augmentation (tea), visual and olfactory augmentation (chocolate) and visual and olfactory augmentation (tea) ). We captured images of a chocolate cookie and a tea cookie, and used these to overlay onto real cookies. The experiment was conducted with 15 participants. The participants had never received training in the anatomy of tastes. And we did not inform the participants beforehand that our system aimed at changing the perceived taste before the participant finished the experiment.
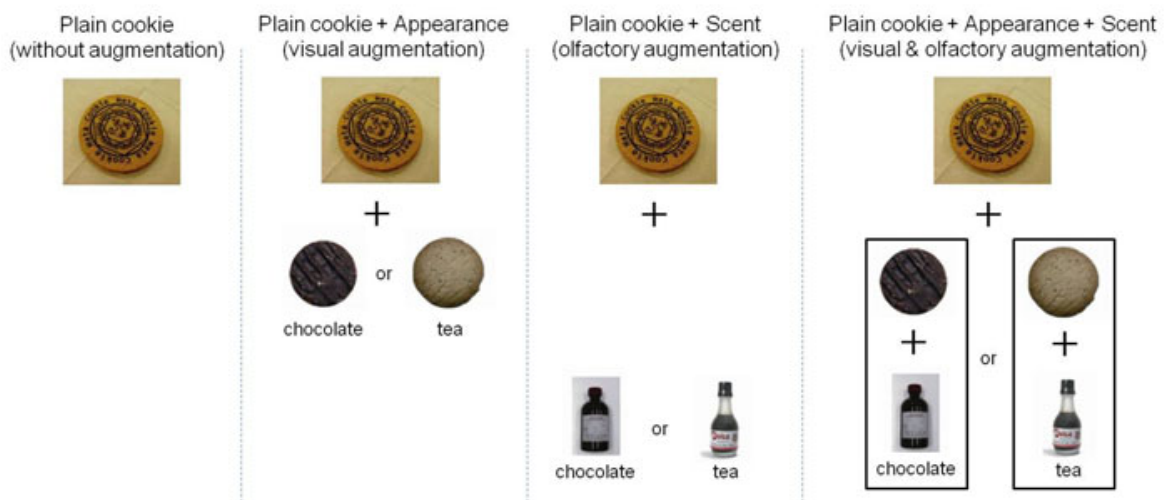

Fig. 6. Experimental conditions

After subjects had eaten the plain cookie and the cookie which was augmented in one of the seven experimental conditions, they were asked to compare it with the plain cookie and to plot their experience of the taste on plotting paper. The plotting paper had two scales from -4 to 4 : one for sweetness and one for bitterness. We defined the origin (0) of the scale as the taste of the plain cookie. Moreover, they were asked to write the taste they identified from the cookie. Subjects repeated these steps 7 times. To eliminate any effect of the order in which the cookie were eaten, the order was randomly assigned by the experimenters. In addition, subjects drank water in the intervals between their eating of the cookies. 


\subsection{Result}

Fig. 7 illustrates the results of this experiment. When the participants ate olfactory augmented cookie, they experienced a change in the cookie's taste in $80 \%$ of the trials. Moreover, when the participants ate cookie with visual stimuli (chocolate) and olfactory stimuli (chocolate), they identified it as the chocolate cookie in $67 \%$ of the trials. And when the participants ate cookie with visual stimuli (tea) and olfactory stimuli (tea), they identified it as the tea cookie in $80 \%$ of the trials. While when the participants ate cookie only with olfactory stimuli (chocolate), they identified it as the chocolate cookie in $47 \%$ of the trials. And when the participants ate cookie with visual stimuli (tea) and olfactory stimuli (tea), they identified it as the tea cookie in $67 \%$ of the trials. These averages in condition of only with olfactory stimuli are lower than the averages in condition of with visual and olfactory stimuli.
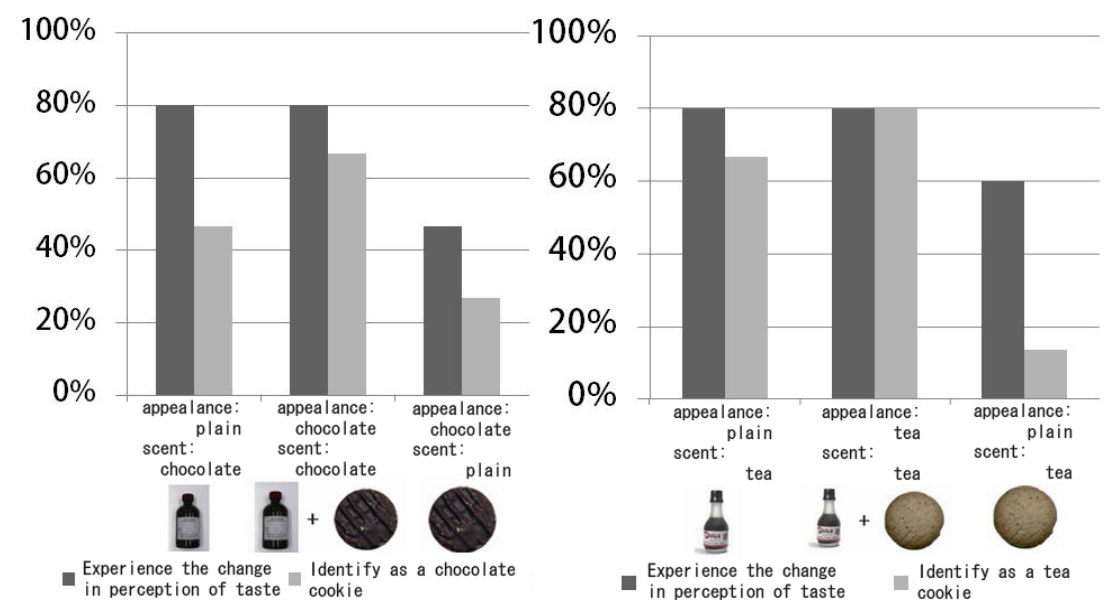

Fig. 7. The cross-modal effect of visual stimuli, olfactory stimuli and visual \& olfactory stimuli on perception and identification of taste

\subsection{Discussion}

The results suggested that olfactory stimuli play an important role in the perception of taste. While the results also suggested that olfactory stimuli cannot change the identification of the taste sufficiently without help of visual stimuli. These do suggest that cross-modal integration among visual, olfactory and gustatory plays important roles for pseudo-gustatory system and our system can change a perceived taste, and lets users experience various flavors without changing the chemical composition by only changing the visual and olfactory information.

\section{Conclusion}

In this study, we proposed a "Psuedo-gustation" method to change the perceived taste of a cookie when it is being eaten by changing its appearance and scent with 
augmented reality technology. We built a "Meta Cookie" system based on the effect of the cross-modal integration of vision, olfaction, and gustation as an implementation of the proposed method. We performed an experiment that investigates how people experience the flavor of a plain cookie by using our system. The results of the experiment suggested that our system can change the perceived taste.

Because our system can shift the flavor of nutritionally controlled foods from distasteful or tasteless to tasty or desired, we believe that it can be used for food prepared in hospitals and in diet food applications. Moreover, we believe we can build an expressive gustatory display system by combining this pseudo-gustation method based on cross-modal integration and methods for synthesizing a rough taste from fundamental taste substances. By doing so, we can realize a gustatory display, which is able to display a wide variety of tastes.

Acknowledgement. This research was partially supported by MEXT, Grant-in-Aid for Young Scientists (A), 21680011, 2009.

\section{References}

1. Nakamoto, T., Minh, H.P.D.: Improvement of olfactory display using solenoid valves. In: Proc. of IEEE VR 2007, pp. 179-186 (2007)

2. Iwata, H., Yano, H., Uemura, T., Moriya, T.: Food Simulator: A Haptic Interface for Biting. In: Proc. of IEEE VR 2004, pp. 51-57 (2004)

3. Maynes-Aminzade, D.: Edible Bits: Seamless Interfaces between People, Data and Food. In: ACM CHI 2005 Extended Abstracts, pp. 2207-2210 (2005)

4. Delwiche, J.: The impact of perceptual interactions on perceived flavor. Food Qual. Prefer. 15, 137-146 (2004)

5. Chandrashekar, J., Hoon, M.A., Ryba, N.K., Zuker, C.S.: The receptors and cells for mammalian taste. Nature 444, 288-294 (2006)

6. Auvray, M., Spence, C.: The multisensory perception of flavor. Consciousness and Cognition 17, 1016-1031 (2008)

7. Rozin, P.: Taste-smell confusion and the duality of the olfactory sense. Perception and Psychophysics 31, 397-401 (1982)

8. Stevenson, R.J., Prescott, J., Boakes, R.A.: Confusing Tastes and Smells: How Odours can Influence the Perception of Sweet and Sour Tastes. Chem. Senses 24(6), 627-635 (1999)

9. Zampini, M., Wantling, E., Phillips, N., Spence, C.: Multisensory flavor perception: Assessing the influence of fruit acids and color cues on the perception of fruit-flavored beverages. Food Quality \& Preference 18, 335-343 (2008)

10. Spence, C., Levitan, C., Shankar, M., Zampini, M.: Does Food Color Influence Taste and Flavor Perception in Humans? Chemosensory Perception 3(1), 68-84 (2010)

11. Ozuysal, M., Calonder, M., Lepetit, V., Fua, P.: Fast keypoint recognition using random ferns. IEEE Transactions on Pattern Analysis and Machine Intelligence 32, 448-461 (2010)

12. Chum, O., Matas, J.: Matching with prosac-progressive sample consensus. In: Proc. of CVPR 2005, vol. 1, pp. 220-226. IEEE, Los Alamitos (2005)

13. Li, L., Huang, W., Gu, I., Tian, Q.: Foreground object detection from videos containing complex background. In: Proceedings of the Eleventh ACM International Conference on Multimedia, p. 10. ACM, New York (2003)

14. Master Mind, Food Printer "Couver", http://www.begin.co.jp/goods/11_plotter/main_01.htm 